\title{
MASSOTHERAPIE MIT BÄLLEN: GESCHICHTE UND ANWENDUNGSTECHNIK
}

\section{REVIEW ARTICLE}

BESSA, Vicente Alberto Lima ${ }^{1}$

BESSA, Maria Fátima de Sousa ${ }^{2}$

BESSA, Vicente Alberto Lima. BESSA, Maria Fátima de Sousa. Massotherapie mit Bällen: Geschichte und Anwendungstechnik. Revista Científica Multidisciplinar Núcleo do Conhecimento. 04-Jahr, Ed. 10, Band 02, S. 05-15. Oktober 2019. ISSN: 2448-0959,

Zugangslink: https://www.nucleodoconhecimento.com.br/gesundheit/massotherapiemit-kugeln

\section{ZUSAMMENFASSUNG}

Massotherapie wurde am Logo der Existenz der Menschheit für verschiedene Zwecke verwendet und es gibt verschiedene Arten von Massagen von klassisch bis transzendental. Unter den verschiedenen Massagemodalitäten gibt es Massage mit Bällen. Es ist eine sehr effiziente Massage und wenig in der aktuellen Literatur angesprochen, so dass die vorliegende Studie durchgeführt wurde und zielte darauf

1 Master in Human Motricity Sciences; Spezialisierung in Dermato-funktionelle Physiotherapie; Spezialisierung auf Sporttraining und Personal Trainner; Spezialisierung in Medizinische Gymnastik; Spezialisierung auf fortgeschrittene Themen in Physiotherapie; Spezialisierung auf Portugiesisch; Spezialisierung in Innovativer Lehre der Hochschulbildung; Absolvent der Physiotherapie; Technologe für Ästhetik und Kosmetik; Bachelor und Bachelor in Leibeserziehung; Graduierte in Briefen; Studium der Pädagogik.

${ }^{2}$ Master in Human Motricity Sciences; Spezialisierung in Medizinische Gymnastik; Absolvent der Physiotherapie; Bachelor und Bachelor in Leibeserziehung. 
$a b$, den Ursprung dieser Massage zu untersuchen und die Anwendungstechniken zu beschreiben. $\mathrm{Zu}$ diesem Zweck wurde eine bibliographische Forschung auf der Grundlage von Büchern und Datenbanken von Scielo und Scholar Google durchgeführt. Es wurde festgestellt, dass diese Art der Massage in der Ming-Dynastie durchgeführt wurde und zusätzlich zur Massage Übungen mit den Bällen durchgeführt werden konnte. Es gibt im Grunde vier Massagetechniken wie Bälle.

Stichworte: Massage, Massage-Vorteile, Massage als Bälle.

\section{EINFÜHRUNG}

Eine der ältesten Therapien, die bisher verwendet wurde, ist Massotherapie und es hat verschiedene Enden wie verminderte Spannungen, Freisetzung von Toxinen, Förderung der Muskelentspannung, Bekämpfung von Müdigkeit und Regenung der Durchblutung. (CANNECCHIA, 2019).

Massage ist eine gängige Praxis in der Geschichte der Menschheit und wurde in der Literatur weit verbreitet. Heute gibt es mehrere Videos der Massotherapie, die leicht über das Internet, auf Facebook, Instagram, YouTube, WhatsApp, etc. abgerufen werden können. Es ist jedoch notwendig, darauf hinzuweisen, dass nicht alles, was in digitalen Medien gepostet wird, korrekt ist und dies für Laien und Anfänger in der Welt der Ästhetik eine Menge Verwirrung schafft. Daher ist es wichtig, Studien zu entwickeln, die die richtige Offenlegung der verschiedenen Techniken der Massotherapie und ihrer tatsächlichen Vorteile ermöglichen.

Es ist bekannt, dass es verschiedene Arten der Massotherapie gibt, wie: Schwedisch, Thai, Hawaiianisch, Tantric, Indian, etc., aber eine der am wenigsten beschriebenen Techniken ist Massage mit Bällen, oder einfach Massage mit Bällen. Es ist eine Massagetechnik, die auf Weichgewebe angewendet wird und drei grundlegende Effekte ausübt: mechanische, physiologische und psychologische. Seine Anwendung folgt Prinzipien ähnlich wie schwedische Massotherapie, jedoch, mit Bällen, um die Körperregion zu massieren. 
Es ist bekannt, dass es einen Mangel an wissenschaftlicher Literatur über die Verwendung von Ballmassage, so dass die vorliegende Studie durchgeführt wurde und zielte darauf $a b$, den Ursprung dieser Massage zu untersuchen und die Anwendungstechniken zu beschreiben. Zu diesem Zweck wurde eine bibliographische Forschung auf der Grundlage von Büchern und Datenbanken bei Scielo und Scholar Google durchgeführt und hatte als Deskriptoren: Massage, Vorteile der Massage, Massage als Bälle.

\section{LITERATURKRITIK}

\subsection{MASSAGE GESCHICHTE}

Es gibt historische Aufzeichnungen, die beweisen, dass sogar die ersten wilden Zivilisationen, zusätzlich zu den zivilisierten, bereits eine Art von Massage praktizierten, vor allem Reibung (WOOD und BECKER, 2008). Es war eine gängige Praxis in alten indischen, chinesischen, japanischen, griechischen und römischen Zivilisationen. Es ist bekannt, dass eine der ältesten Referenzen in Nei Ching erscheint, einem chinesischen medizinischen Text, der in einer Zeit vor 1500 v. Chr. geschrieben wurde. Später wurden Schriften über Massagen von Hippokrates Ärzten im 5. Jahrhundert v. Chr. gefunden. und Avicenna und Ambrosius Stop, im 10. und 16. Jahrhundert. (CASSAR, 2001).

Jedoch, Ägypter bereits Massage für therapeutische Zwecke auf mehr als 4000 a angewendet. C. Und in Indien suchte die Massage eine ganzheitliche Vision, um Körper, Geist und Seele in Einklang zu bringen und eine der Empfehlungen und Lehren zu sein. Es ist bekannt, dass die Beschreibung der Massage in den Büchern Ayur Veda liegt, die um 1800 v. Chr. geschrieben wurden. Es ist jedoch bekannt, dass fast alle großen Kulturen der Welt im Detail die Indikationen und Vorteile der Massage vorgeschlagen haben, die häufig mit anderen Arten der traditionellen Behandlung kombiniert wurde, insbesondere Badbehandlungen. (SILVA, 2016).

Es ist notwendig, hervorzuheben, dass Hippokrates (480 v. Chr.) die Massage der Anatripe nannte, deren Bedeutung "Reiben des Gewebes" ist, später wurde der Begriff 
in das lateinische Wort frictio übersetzt, was "Reibung" oder "Reiben" bedeutet. Es ist bekannt, dass dieser Begriff lange Zeit vorherrschte und in den Vereinigten Staaten bis 1870 verwendet wurde. In Indien wurde die Massage Shampoing genannt; in China, Cong-Fou; in Japan, Ambouk (CASSAR, 2001).

Hippokrates (460-370 v. Chr.) wiederum nutzte Die Massage als Behandlung, weil er glaubte, dass der Körper in der Lage ist, sich selbst zu heilen, wenn er richtig stimuliert wird. Ein weiterer griechischer Arzt, der Massagen, Diäten und Übungen zur Behandlung von Krankheiten einsetzte, war ein weiterer griechischer Arzt. Darüber hinaus ist es berüchtigt zu wissen, dass die primitiven australischen, ägyptischen, russischen, ukrainischen, pazifischen und nord- und südamerikanischen Zivilisationen Massage durch Reibungsbewegungen mit Ölen und Wasser als eine Möglichkeit, Dämonen und Geister, die Krankheiten bei Menschen verursachten und so den Körper der Kranken reinigten. Daher haben mehrere Menschen verwendet und empfohlen Massage, um die Gesundheit des Menschen zu nutzen. (BERTOJA e TOKARS, 2018).

Allerdings wurde die Massage nicht immer gut angesehen, mit dem Aufstieg des Christentums im Mittelalter (die historische Periode vom 5. Jahrhundert bis zum 15. Jahrhundert), wurde der Kult des Körpers als Sünde betrachtet. Daher wird hygienenicht mehr gepflegt und daher war massage in Crested Zivilisationen verboten. (BRAUN und SIMONSON, 2007). In Europa betrachtete die katholische Kirche Massage als Sünde und ihre Assoziation mit Heilkräutern wurde als ein Akt der Hexerei angesehen. (RAMOS, 2017).

Mit dem Ende des Mittelalters und der Entstehung der Renaissance wurde die Massage entdeckt und wieder angewendet, und mehrere Autoren trugen dazu bei. Zwischen 1776 und 1839 revolutionierte Pehr Henrik Ling jedoch die Praxis der Übungen und Massagen. Er schuf die Terminologien und Technik der schwedischen Massage, die auch als klassische Massage oder Heilung durch schwedische Bewegung bekannt ist. Seine Technik wurde zunächst in Europa und Russland populär gemacht und später globalisiert (WOOD und BECKER, 2008). 
Es war auch Hippokrates, der die Vorteile der Massage im Zusammenhang mit den chemischen Eigenschaften der verwendeten Öle beschrieb (FRITZ, 2002). Daher kann man sehen, dass nicht nur Massage Vorteile bringen könnte, sondern auch die chemische Eigenschaft bestimmter pflanzlicher und ätherischer Öle.

Das Wort Massage stammt aus der griechischen Masse, die "Kneten" bedeutet, was Berührung, Handhabung, Anziehen bedeutet (FRITZ, 2002). Allerdings ist der Begriff Massotherapie derzeit vorzuziehen, da das Wort Therapie auf die therapeutische Anwendung von Massagen hinweist. Heutzutage gibt es mehrere Massagetechniken unter innen: Entspannen, Sport, therapeutische, Modellierung, Schnellmassage, reflexologisch, tantrisch, etc. Genauso wie mehrere Arten von Geräten entstanden sind, die dem Therapeuten helfen, die Massage anzuwenden, sind darunter die Baoding-Bälle () oder Gesundheitsbälle.

Die Baoding-Kugeln (Abbildung 1) sind chinesischer Herkunft und ihre Bezeichnung stammt aus der Stadt baoding, die sich in der Provinz Hebei in der Volksrepublik China befindet. Sie sind Artefakte verwendet, um Hände zu trainieren und sorgen entspannung, Verringerung der Belastung. Traditionell bestehen sie aus einem Paar kleiner Eisenkugeln, die in einer kleinen rechteckigen Box gelagert werden. Diese Kugeln stammen aus der Ming-Dynastie (1368-1644 n. Chr.) und wurden von einem Waffenhandwerker hergestellt, der von einem himmlischen Traum geleitet wurde. Er schuf zwei Eisenkugeln, die auf dem Yin/Yang-Konzept basierten: eine, die das Drachengebrüll darstellt, und die andere die Ecke des Phönix.

Zunächst war der Kaiser von China von den therapeutischen Vorteilen der Bälle bezaubert und machte sie zu einem Instrument, das nur von der königlichen Familie verwendet werden konnte. Später wurden Eisenkugeln populär und erreichten die Welt. Anfangs wurden sie verwendet, um die körperliche Verfassung zu verbessern und das Temperament zu trainieren (Angst verringern, beruhigen) Zweck, für den sie noch heute verwendet werden. Sie sind ein Apparat, um Muskeln und Gelenke zu entspannen und zur Verteidigung in Derierkunst-Praxis. 
Heutzutage sind Baoding-Kugeln aus mehreren Arten von Materialien hergestellt, bleiben aber leicht zu tragen. Sie sind meistens hohl und aus Chromstahl gefertigt und enthalten Guizos im Inneren. Die Guizos liefern Klänge, die wie entspannende Glocken klingen können, die auf die Natur und den Einfluss der fünf Finger reagieren, so Muskeln trainieren und die Durchblutung aktivieren.

Abbildung 1: Bälle baoding.

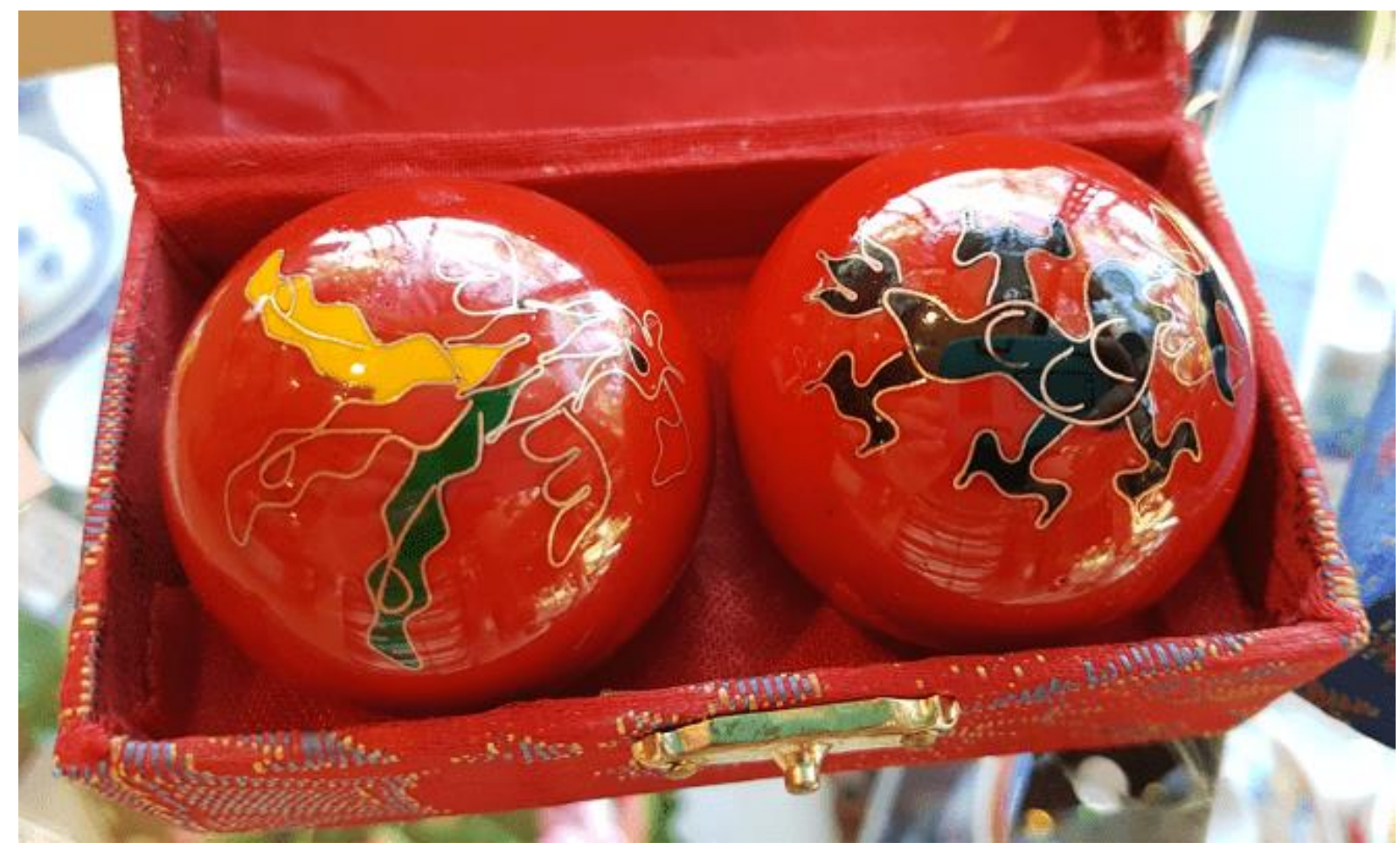

Fonte: Os autores

Mit der Entwicklung neuer Materialien und der Entwicklung der Technik begannen die Kugeln mit magnetischen Materialien, Holz, Silikon und Metall, Gummi, Silikon usw. hergestellt zu werden. (Abbildung 2) 
Abbildung 2: Verschiedene Arten von Kugeln.
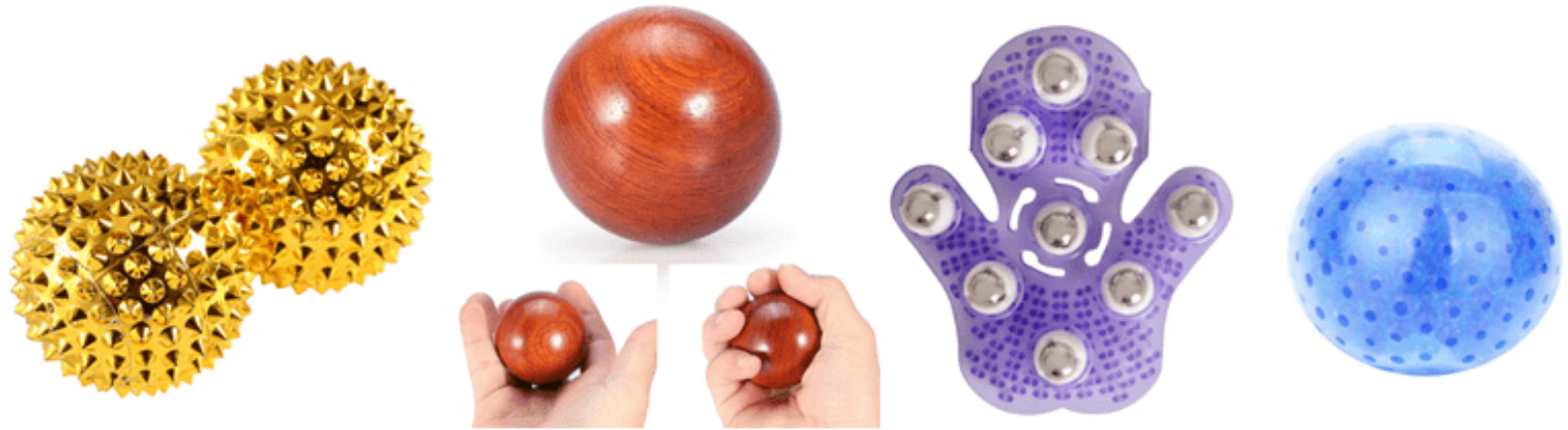

Fonte: Os autores

Als die eisernen Kugeln auftauchten, waren sie einzigartig, um ihre Hände zu massieren. Die Person selbst massiert, indem sie die Bälle zwischen seinen Fingern dreht. Diese Technik ist immer noch weit verbreitet, aber es ist möglich, alle Teile des Körpers mit Hilfe des Therapeuten oder sogar verschiedene Teile des Körpers durch Selbstmassage zu massieren. (Abbildung 3)

Abbildung 3: Selbstmassage mit Ball.
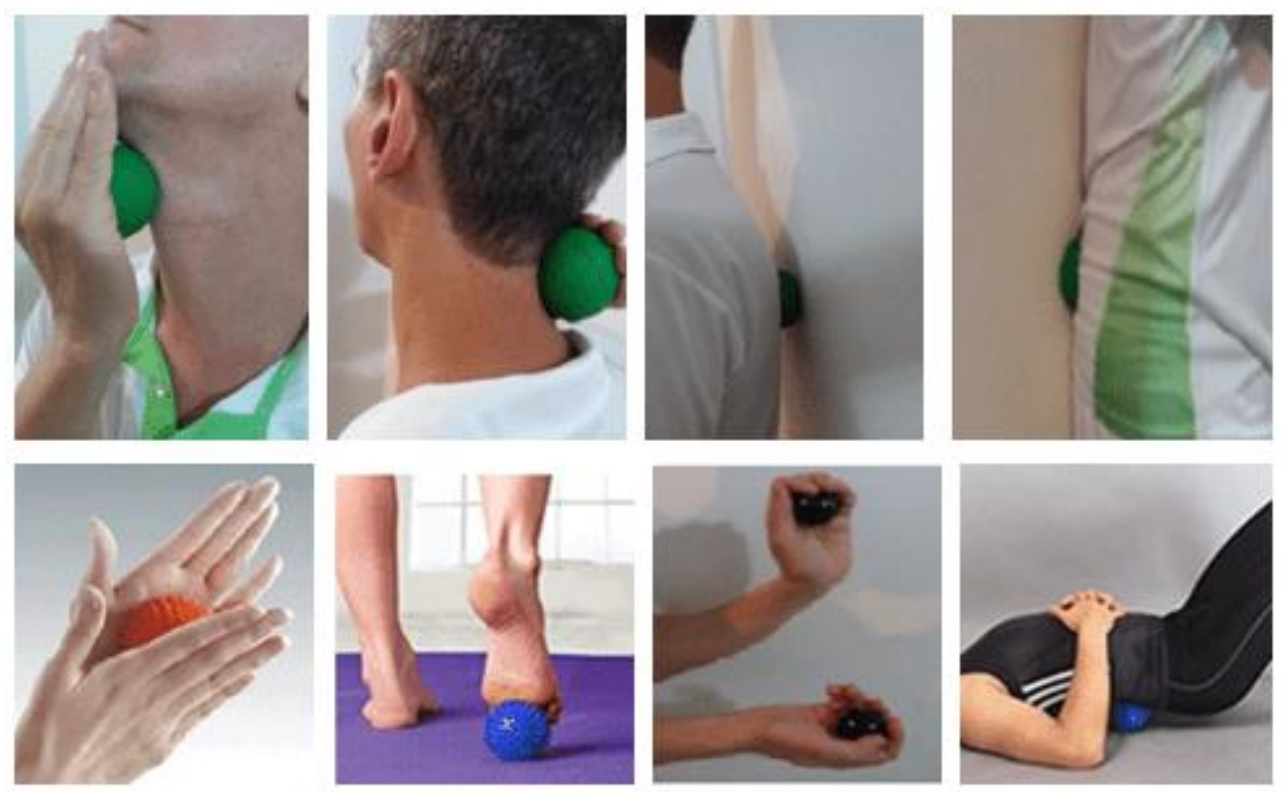

Fonte: Os autores 


\subsection{BALLMASSAGE: VORTEILE}

Die Ballmassage (Ballmassage) wird auf Weichgewebe angewendet und übt drei grundlegende Effekte aus: mechanische, physiologische und psychologische. Die Haupteffekte der Massage sind mechanisch, aber sie erzeugen physiologische und psychologische Effekte.

Massage ist eine Technik, die Manipulation auf Weichgewebe fördert und wie jede Art von entspannender Massage, ermöglicht es die Verringerung der Muskelverspannungen, zusätzlich zur Stimulierung der Produktion von Serotonin und Histamin, die Vasodilatator Wirkung haben, Erhöhung Blut- und Lymphintinz. Massage kann die Rmorezeptoren aktivieren und die Durchlässigkeit der Zellmembran erhöhen, dies ermöglicht Nährstoffaufnahme und damit eine bessere Zellernährung, Sauerstoffversorgung und Hydratation (CLAY, 2008).

Psychologisch ist die Ballmassage in der Lage, Muskel- und mentale Entspannung zu bieten, was zu einem anfälligen Zustand zum Schlafen führt. Es ist bekannt, dass Massage stimuliert die Freisetzung von Endorphinhormonen und Serotonin, die für das Gefühl der Freude verantwortlich sind. (PEREIRA, 2013). Endorphinhormone und Serotonin ermöglichen Entspannung und sind gleichzeitig diejenigen, die für den Tiefschlaf verantwortlich sind, der mit REM-Schlaf bezeichnet wird. Darüber hinaus reduziert Massage Cortisol, das einer der Verantwortlichen für Schlaflosigkeit/Stress ist, so dass eine Person, die die Massage erhält, weniger Cortisol in der Blutbahn hat, was zu einer Verbesserung inrer Schlafqualität und zur Verringerung von Angst und Stress führen wird.

Die Dehnungs-, Kompressions-, Traktions- und Reibungsbewegungen, die bei der Ballmassage durchgeführt werden, üben offensichtliche mechanische Kräfte auf das Gewebe aus. Diese mechanischen Kräfte üben mobilisierende Effekte der Erweichung und Dehnung auf die Haut, subkutane Gewebe, Eingeweide und Muskeln.

Der mechanische Effekt bezieht sich auf die direkten Einflüsse, die Massage auf Weichgewebe spielt, die massiert werden (CASSAR, 2001). Es bietet physiologische 
Auswirkungen auf Muskel, Nerven, Kreislauf und Verdauungssysteme. Massage mit Bällen beim langsamen Entfernen der Muskeln stimuliert den Sehnenreflex, der Muskelverspannungen reduziert und $z u$ Muskelentspannung führt. Mit Skelettmuskelentspannung und Gleitmanövern mit Bällen ist es möglich, genügend Druck auszuüben, um die Flüssigkeiten in Richtung venöser oder lymphatischer Kreislaufströmung zu verdrängen. Wenn Manöver kräftig sind, können sie zu signifikanten Erhöhungen des Blutflusses aufgrund von Vasodilatations- und Kapillarisierungsphänomenen führen. Diese Kreislaufeffekte fördern den Austausch zwischen Zell- und Blutmedien, verbessern den Sauerstoff- und Nährstoffeintrag, helfen bei der Beseitigung von Rückständen aus Stoffwechsel und Kohlendioxid. Manöver mit mehr Suable-Druck, rhythmisch und auf die Pfade der oberflächlichen Lymphgefäße angewendet ermöglichen es, den lymphatischen Rückfluss zu beschleunigen, nützlich in Fällen von Ödemen, um bei der Resorption zu helfen.

Die wichtigsten mechanischen Effekte, die durch Massagen erzeugt werden, sind: die Mobilisierung von venösem Blut, Lymphe, Ödemen und Hämatomen, Darmgehalt, Schleim, Muskelfasern und Masse, Sehnen, Haut und Unterhautgewebe, Adhäsionen und Narbengewebe (Staatsregierung der Cearé, 2010).

Wir heben auch die physiologischen Auswirkungen der Ballmassage auf das Verdauungssystem hervor, die den Darmtransit verbessern. Massage stimuliert Peristaltik, um die Evakuierung von Wohnungen und Kot aus dem Dickdarm zu fördern, Erleichterung der Ausscheidungsfunktion in Fällen von Darmverstopfung.

Zweifellos wird die Verringerung der Schmerzen bei der Anwendung der Massage durch verschiedene Mechanismen erhalten, unter der Abnahme der Muskelkrämpfund und Ödeme. Daher absorbiert die Verbesserung der Kreislaufzirkulation Ödeme und damit die zellulären Stoffwechsel. Es reduziert auch durch die Aktivierung von Hautrezeptoren, die Schmerzen durch den Mechanismus des Tores hemmen (STARKEY, 2017).

Muskelentspannung und verbesserte Durchblutung tragen bereits zur Verringerung der Muskel-Skelett-Algie bei. Allerdings reduziert Die Ballmassage Schmerzen, weil 
sie den Phänomenen der Hochwassertheorie gehorcht. Diese Theorie erlaubt es uns zu verstehen, dass es verschiedene Arten von Nervenfasern gibt und jede hat eine Geschwindigkeit der Durchführung nervöser Impulse und eine Funktion. In diesem Fall ist die Schmerznervenfaser langsamer als Takt- und Drucknervenfasern. Wenn also eine Person einen Muskelschmerz verspürt und massiert wird, kommen Nervenimpulse der Berührung und des Drucks zuerst im Rückenmark an und hemmen die Nervenbahnen von Schmerzen, die Schmerzinformationen zum Thalamus (Der Ort des Gehirns, der es ermöglicht, die r).

Die Wirkung der Massage auf die Schmerzkontrolle ist so wichtig, dass sie als Palliativmedizin bei Patienten mit fortgeschrittenem Krebs stadiumiert. Eine Tatsache, die in florentine Aussage (2012, n.p.) gefunden wird, die berichtet, dass "Massage eine Technik ist, die als Komplementärtherapie bei Krebspatienten verwendet wird, mit dem Ziel, Schmerzlinderung zu bieten."

Es sollte betont werden, dass für hemmende Effekte, ist es notwendig, dass Massagemanöver mit Bällen ständig, langsam und reibungslos durchgeführt werden, so dass die Anpassung durch Erhöhung der Schwelle der Wahrnehmung des neuen Reizes erfolgt.

Die Ballmassage hilft bei der Erneuerung der epidermalen Zellen und ermöglicht es, die Talgdrüsen zu klären und besser zu arbeiten. Und durch Vasodilatator-Phänomene und Kapillarisierung wird die Haut mehr genährt und sauerstoffrundiger, neben der Erleichterung der Durchlässigkeit einiger kosmetischer Vermögenswerte.

Massotherapie kann einen Einfluss auf das Immunsystem ausüben, da es auf die Hautschichten wirkt, die positiv reagieren. (CARVALHO e ALMEIDA, 2018).

\subsection{BALL MASSAGE: ORIENTIERUNGEN, INDIKATION UND KONTRAINDIZIEN}

Vor der Anwendung der Massage mit Bällen ist es notwendig, dass der Therapeut eine Bewertung (Anamnese) des Klienten. Die Auswertung wird nützliche Informationen 
liefern, um zu wählen, ob die Person zu massieren oder nicht, und ermöglicht es, die prioritätsären Manöver anzuwenden. Wenn der Klient während der Bewertung eine relative Kontraindikation vorlegt, sollte er angewiesen werden, einen anderen Arzt zu konsultieren und kann nur massiert werden, wenn er eine schriftliche Genehmigung des Arztes oder Physiotherapeuten hat.

Die wichtigsten Daten, die im Bewertungsformular enthalten sein sollten, sind: Identifikationsdaten (vollständiger Name, Anschrift, Telefonnummer, Geburtsdatum, Familienstand und Beruf), Daten über den Gesundheitszustand (ob die Person gesund ist oder an Krankheiten leidet: Diabetes, Bluthochdruck oder Hypotonie, Herzerkrankungen, Krebs, Migräne, Epilepsie, Verstopfung, Dysmenorrhoe, Zystitis, Allergien, Schlaflosigkeit, Depression, etc.) und es ist notwendig zu wissen, ob die Person schwanger ist oder stillt. Weitere Informationen, die in der Bewertungsform enthalten sein können, sind die Ausübung körperlicher Bewegung, Fütterung, Konsum alkoholischer Getränke, Tabak und Drogenkonsum.

Die Anwendung der Ballmassotherapie achtet auf die gleichen Kontraindikationen jeder Massageanwendung und sie können absolut sein, das heißt, Massage kann nicht auf den Kunden angewendet werden; oder relativ, das heißt, es ist möglich, Massage anzuwenden, aber einige Pflege sollte angenommen werden oder einige Bereiche können nicht massiert werden.

Die wichtigsten absoluten Kontraindikationen sind Thrombose oder instabile Gefäßschäden, fiebriger Zustand, Vorhandensein von Infektionen, Gangrän, Nierenerkrankungen, fortgeschrittene Herzerkrankungen, starke Kopfschmerzen, unkontrollierte Blutdruckänderungen durch Medikamente, Rausch. Relative Kontraindikationen sind: Frakturen (vor erstarrt), jüngste Verbrennungen, offene Wunden. (VERSAGI, 2015). Andere relative Kontraindikationen sind: Krebs, psychische Erkrankungen, Entkalkungen und Schwangerschaft.

Die wichtigsten Indikationen sind: Stressabbau, Verstopfung, Migräne, Genesung der Person bei traumatischen Verletzungen (Sprain, Prellungen) nach ärztlicher Freisetzung, Verringerung der Muskel- und Sehnenspannungen, Myalgien durch 
Anstrengung, Prävention von STDs, Drainage von venösen und lymphatischen Ödemen, adjuvant bei der Behandlung von Dehnungsstreifen, lokalisierter Lipodystrophie, fibrosklerotischeödem Panikuopathie, Schlaflosigkeit, Angst, etc.

\subsection{BALLMASSAGE: TECHNIKEN}

Einige Grundregeln für die Anwendung der Massage sollten befolgt werden, wie: sowohl der Massagetherapeut und der Klient sollte sich während der Sitzung wohl fühlen; Kontakt mit dem Kunden sollte nicht plötzlich entfernt werden; Massage sollte in Regionen unterbrochen werden, in denen der Patient Schmerzen verspürt; nie direkt auf die Wirbelsäule drücken oder massieren. (ELLSWORTH, 2012).

Ballmassage-Manöver müssen die Prinzipien der Richtung, Druck, Geschwindigkeit, Tempo und Dauer entsprechend den gewünschten Zielen respektieren.

Die Richtung hängt von der gewünschten Wirkung ab, so dass sie in der Zirkulationsrichtung durchgeführt werden kann, die gewünscht wird, um zu steigern oder in Richtung der Muskelfasern, die massiert werden sollen. Was den arteriellen Fluss betrifft, so sollten Manöver Zentrifugen oder nahezu distal sein, um Gliedmaßen, Rumpf und Hals und Zentripetal oder Proximal zu massieren, um den Kopf zu massieren. Wenn die Fließrichtung venös ist, müssen die Manöver zentripetal oder this-proximal sein, um Gliedmaßen, Rumpf und Hals und Zentrifugen zu massieren oder nahezu distal, um den Kopf zu massieren. Wenn auf Lymphdrainage zielt, ist es notwendig, die Richtung des Flusses der Lymphgefäße zu folgen und die Drainagesequenz zu erhalten, die von José Maria Pereira de Godoy und Maria de Fétima Guerreiro Godoy vorgeschlagen wurde.

Wie für den Druck ausgeübt werden, sollte es bequem und angenehm sein, wenn Muskelentspannung und Schmerzlinderung suchen. Bemerkenswert ist, dass kundenfeedback benötigt wird, um den Druck anzupassen. Es gibt mehr muskulöse oder fettleibige Menschen, bei denen der Druck im Vergleich zu schlanken, älteren menschenoder Kindern tendenziell etwas höher ist. Wenn Sie eine Lymphdrainage anstreben, sollte der Druck zwischen 30 und 40 mmHg liegen. 
Geschwindigkeit und Tempo variieren auch je nach Bedarf. Entspannungs- und Entwässerungsmanöver müssen ohne langsam, konstant und gut rhythmisch sein. Für arterielle Kreislaufdynamisierung, Striationsbehandlung, lokalisierte Lipodystrophie, Fibroedemageloid sollte schneller sein. Es ist gut, sich daran zu erinnern, dass Bewegungen, die langsam durchgeführt werden, dazu neigen, entspannend zu sein, während das Gegenteil, anregender ist.

Die Massage dauert von 5 bis 20 Minuten, wenn sie in nur einer Region angewendet wird, außer wenn das Ziel Lymphdrainage ist, in diesem Fall wird es von 30 bis 40 min dauern. Wenn sie systemisch angewendet wird, d.h. im ganzen Körper, die auf Muskelentspannung und verminderte emotionale Spannungen abzielt, dauert es von 45 bis $90 \mathrm{~min}$.

Es sollte beachtet werden, dass die Zeit stark von dem zu erreichenden Ziel und von der Größe des Körperbereichs abhängt, da es weniger Zeit in Anspruch nimmt, eine relativ kleine Person im Vergleich zu einer großen Person zu behandeln. Bei sehr jungen und sehr älteren Menschen sollte die Dauer der Massage reduziert werden, da der Reflexbogen empfindlicher ist und die integrale Wirkung schneller erreicht wird.

Die vom Therapeuten angewendete Ballmassage ist in vier Grundmanöver unterteilt und jedes Manöver hat seine Variationen. Das Manöver, das den Dienst initiiert und beendet, ist das des Gleitens, das aus dem Gleiten zu oder mit den Kugeln auf der Körperoberfläche des Kunden besteht, immer als Reaktion auf den gewünschten Kreislauffluss oder die Richtung der Muskelfasern oder das Gefühl der Peristaltik (Abbildungen 4 bis 11). 
Abbildung 4: Symmetrische Längsdurchlauftechnik in Mmli.

Deslizamento longitudinal simétrico com as 2 bolas no membro inferior
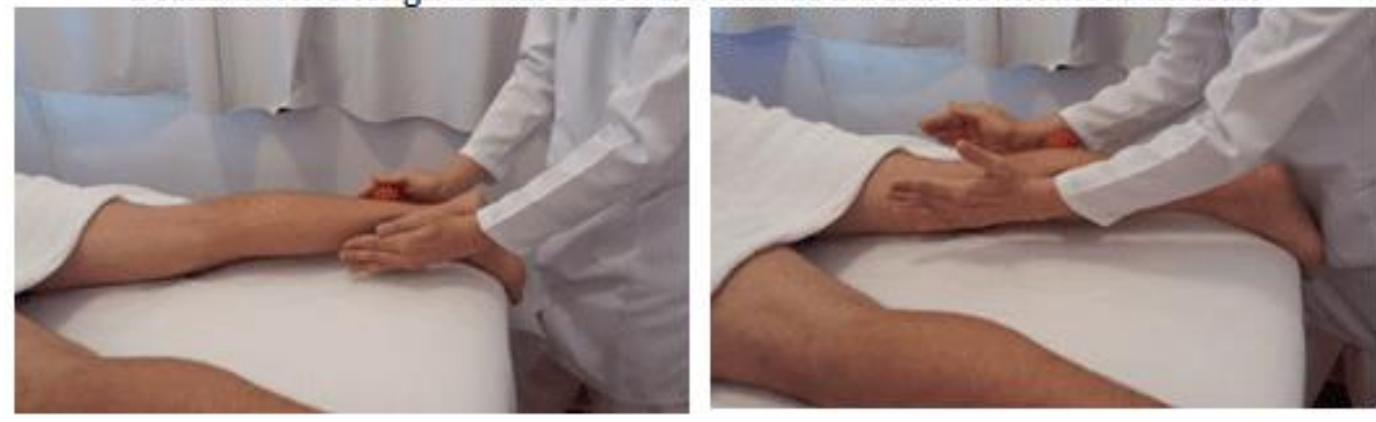

Fonte: Os autores

Abbildung 5: Asymmetrische Längs-Slip-up-Technik im Kofferraum.

\section{Deslizamento longitudinal assimétrico com as 2 bolas na região tóraco-lombar}
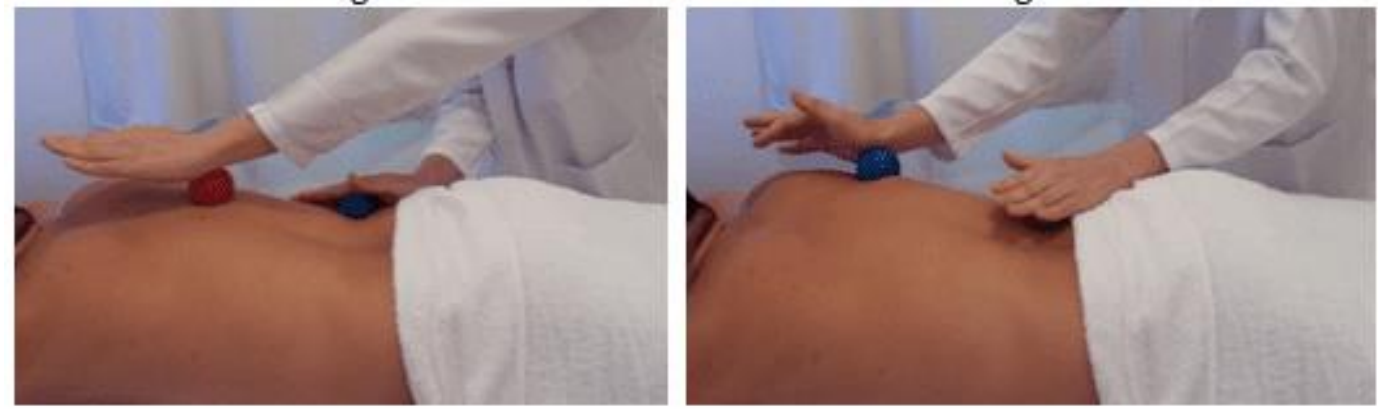

Fonte: Os autores

Abbildung 6: Cross-Slip-Technik.

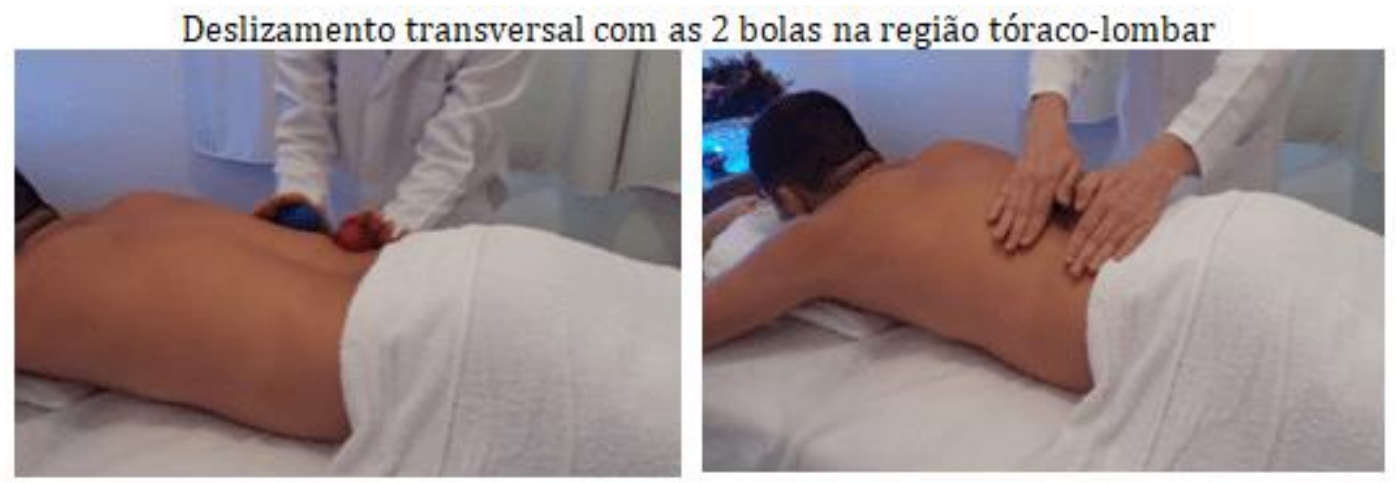

Fonte: Os autores 
Abbildung 7: Symmetrische Längs-Slip-up-Technik im Kofferraum.

Deslizamento longitudinal simétrico com as 2 bolas na região tóraco-lombar
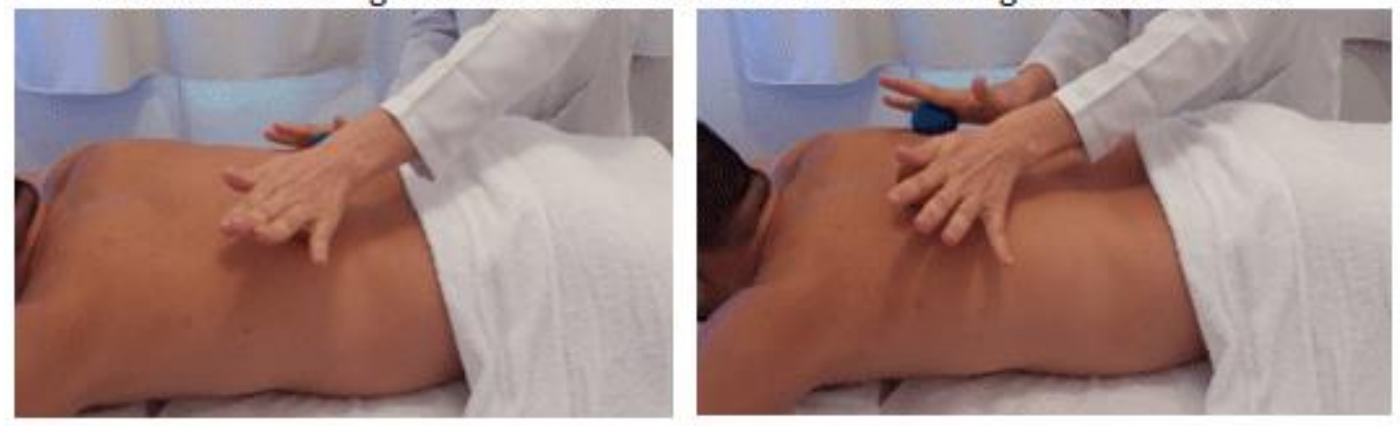

Fonte: Os autores

Abbildung 8: Rundschiebetechnik.

Deslizamento circular com uma bola na região abdominal no sentido do peristaltismo. A manobra pode ser executada com uma ou duas mãos.

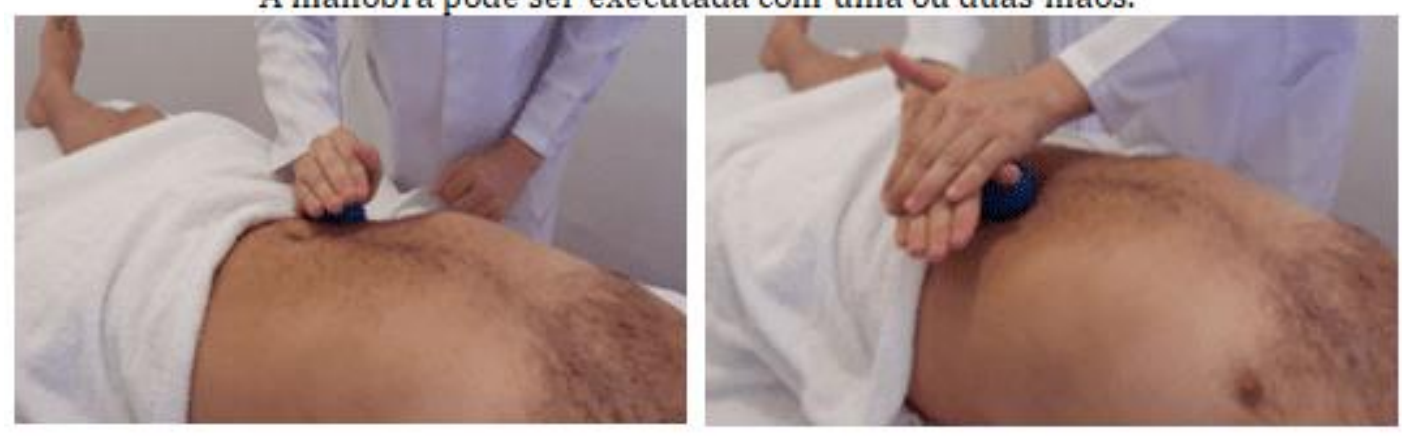

Fonte: Os autores

Abbildung 9: Symmetrische Längs-Slip-up-Technik am Unterarm.

Deslizamento longitudinal simétrico com as 2 bolas na mão e no antebraço
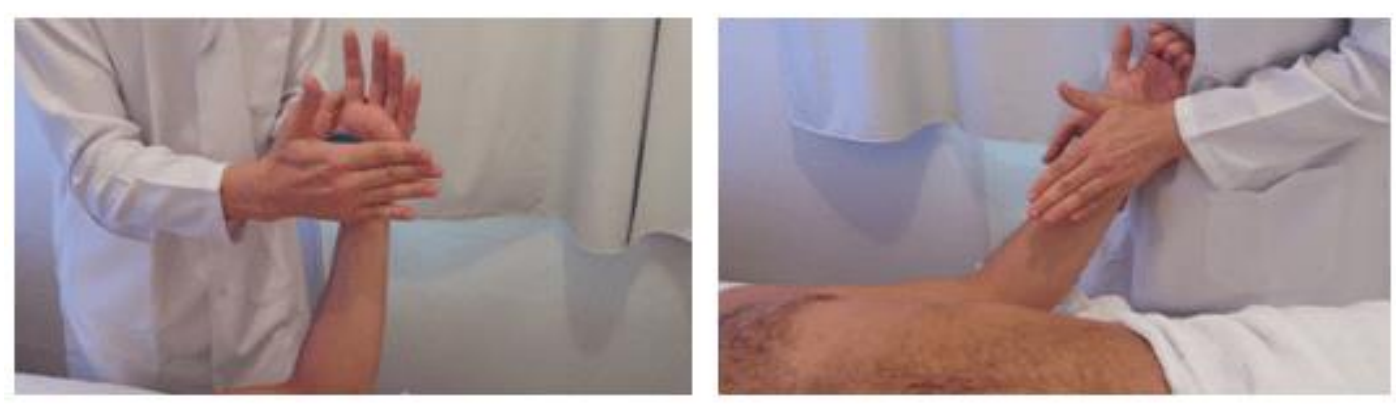

Fonte: Os autores

RC: 41889

Disponível em: https://www.nucleodoconhecimento.com.br/gesundheit/massotherapie-mit-kugeln 
Abbildung 10: Symmetrische Längsschlupftechnik am Arm.

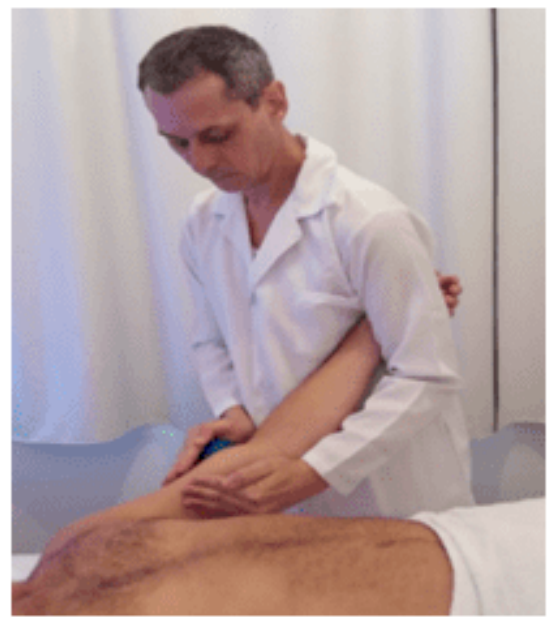

Deslizamento longitudinal simétrico com as 2 bolas no braço. Observe que o terapeuta sustenta o membro superior fixando-o entre o tronco e o braço. É fundamental que o cliente não sustente 0 membro.

Fonte: Os autores

Abbildung 11: Diagonale Gleittechnik.

Deslizamento diagonal com as 2 bolas na região escapular
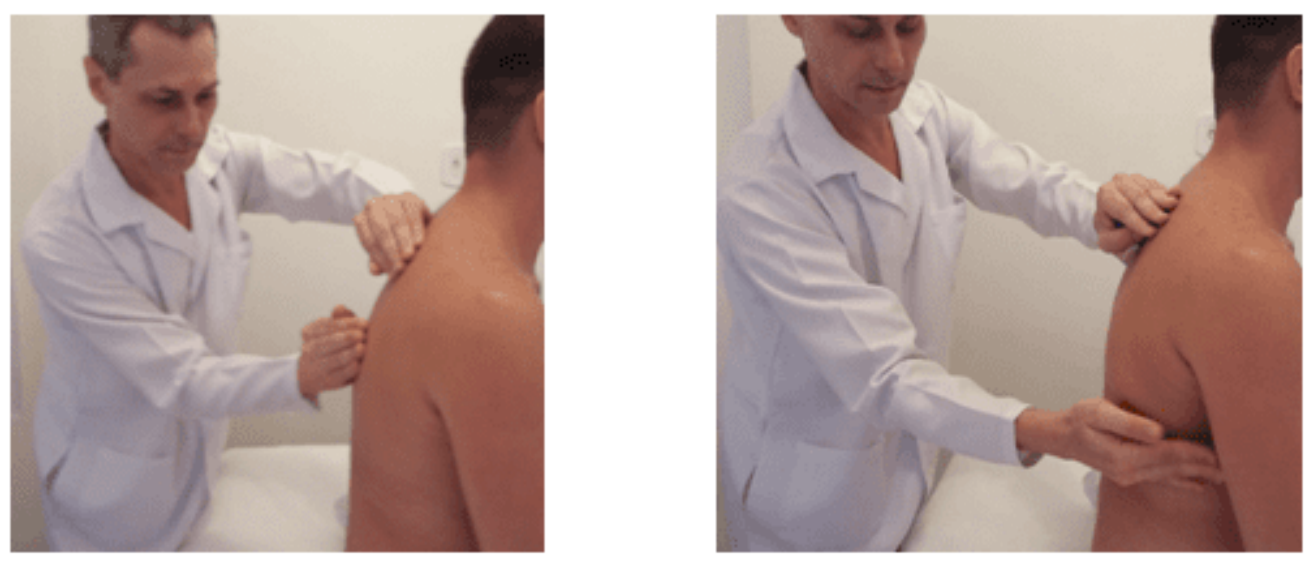

Fonte: Os autores

Das zweite Manöver ist feste Komprimierung oder Komprimierung und Ziehen. Die erste besteht darin, die Region 10 bis 15 Sekunden lang oder während der gesamten Sitzung durch den Ball komprimiert zu halten. Die zweite besteht darin, den Ball gegen den Körper zu komprimieren und in kreisförmige Bewegungen zu ziehen (Abbildungen 12 und 13). 
Abbildung 12: Kompressionstechnik im Fuß.

\section{Compressão fixa e compressão e arrasto com uma bola na região plantar}
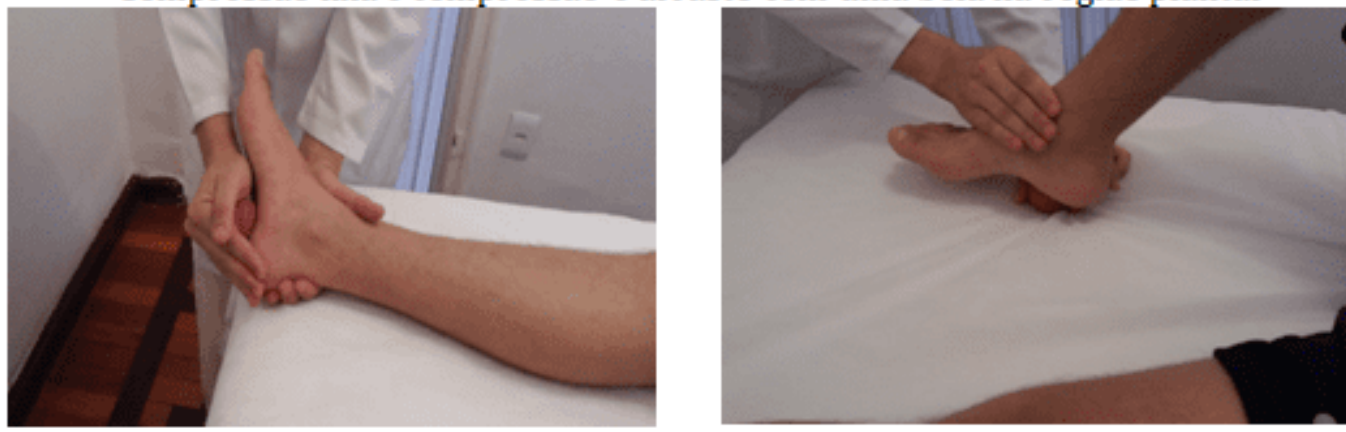

Fonte: Os autores

Abbildung 13: Kompressionstechnik in der Wirbelsäule.

\section{Compressão fixa com as 2 bolas na região paravertebral cervical e lombar}
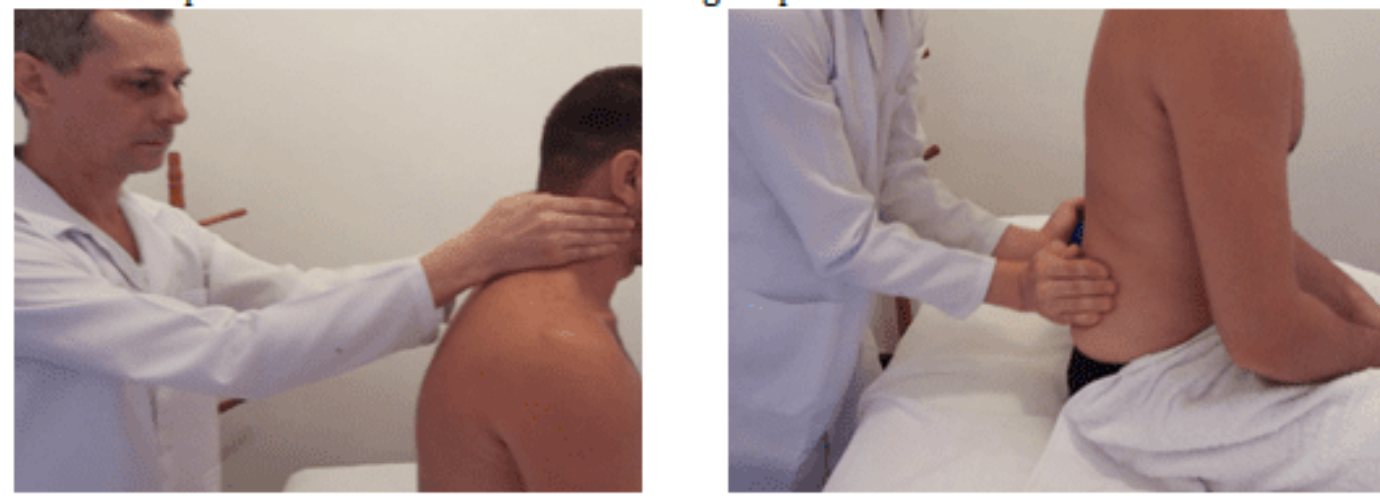

Fonte: Os autores

Das dritte Manöver knetet und in diesem Fall werden immer zwei Kugeln verwendet. Der Therapeut knetet das Weichgewebe zwischen den Kugeln mit schönem Druck oder starkem Druck, aber nicht unbequem. Der Kunde darf keine Schmerzen oder Belästigungen in irgendeiner Hypothese melden (Abbildung 14). 
Abbildung 14: Kneading-Technik.

Amassamento de trapézio e da loja posterior de perna
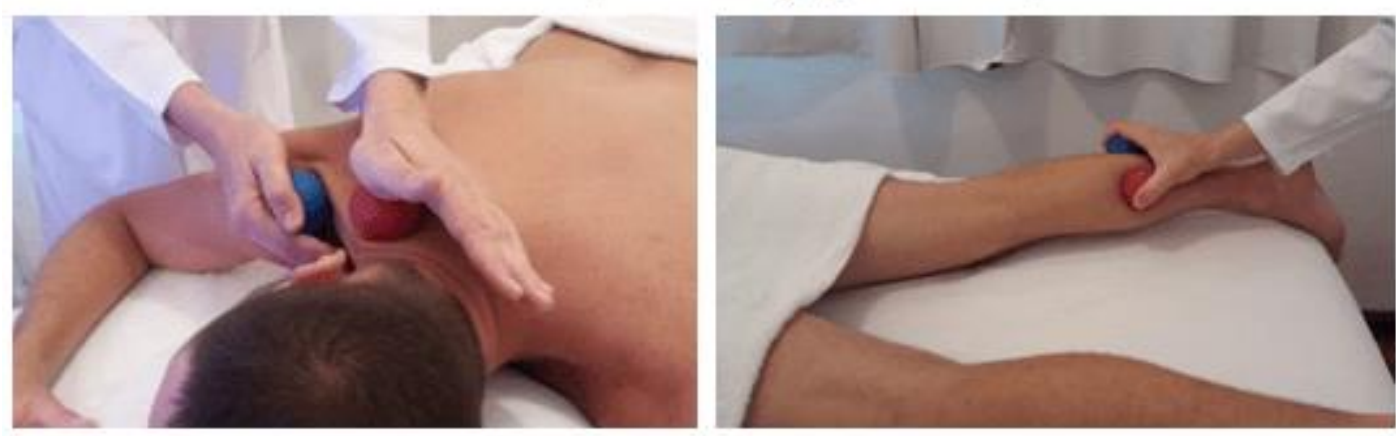

Fonte: Os autores

Das vierte Grundmanöver ist Reibung und in diesem Fall wird es in der Regel in kleinen Regionen und mit nur einer Kugel verwendet. Dieses Manöver ist eine Art tiefen Ausrutscher, der kräftig und schnell durchgeführt wird, um Muskelverspannungen zu reduzieren, Gerinnsel rückgängig zu machen, die lokale Mikrozirkulation zu erhöhen. Verursacht eine große Erwärmung der massierten Region und wird bei Stosten und lokalisierter Lipodystrophie empfohlen. Die Manöver zielen darauf ab, die Region zu erwärmen und werden mit dem Ball durchgeführt, der zwischen der Handfläche und der Körperoberfläche des Kunden zirkuliert (Abbildung 15).

Abbildung 15: Reibungstechnik.

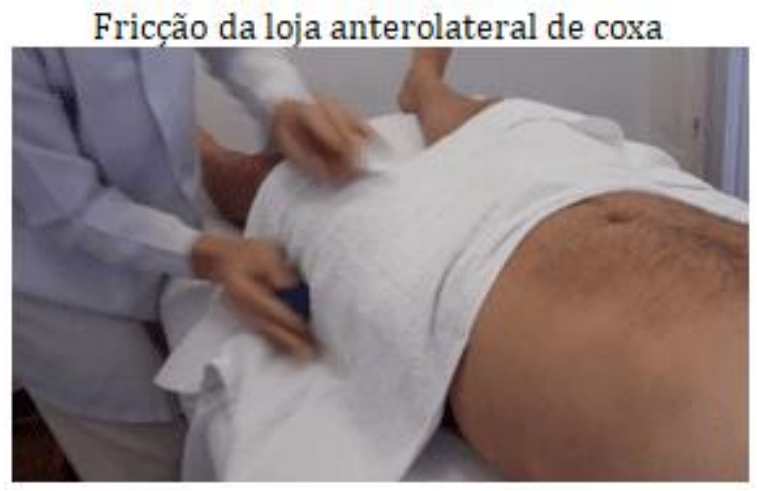

Fonte: Os autores 
Es gibt Variationen von jedem der grundlegenden Manöver, zusätzlich zu der Technik der Selbstmassage mit Bällen, die die Person die Massage auf sie anwendet (Abbildung 16).

Abbildung 16: Selbstmassagetechnik.

\section{Self Ball Massage}

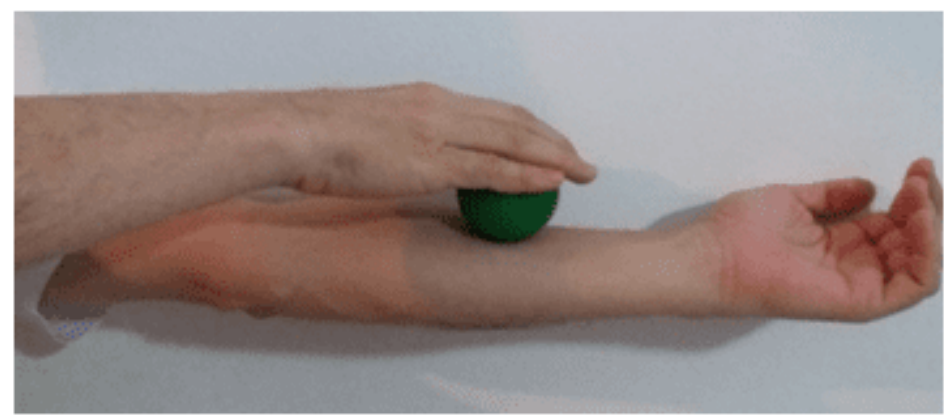

Fonte: Os autores

\section{FAZIT}

Die ersten historischen Aufzeichnungen der Massotherapie stammen aus dem Jahr 1800 v. Chr. und ist in den Büchern Ayur Veda gefunden, aber es ist bekannt, dass die Ägypter Massage für therapeutische Zwecke für mehr als 4000 a verwendet. C. E es war in der Ming-Dynastie (1368-1644 n. Chr.), dass ein Waffenhandwerker zwei Eisenkugeln auf der Grundlage des Yin/Yang-Konzepts schuf. Sie begannen eingesetzt zu werden, um therapeutische Effekte zu bieten und die körperliche Verfassung $z u$ verbessern und temperamentvoll zu trainieren (Angst verringern, beruhigen).

Heute werden noch Eisenkugeln verwendet, aber andere Materialien entstanden mit Holz, Silikon, Gummi und einigen anderen. Alle diese Bälle, unabhängig vom Material, dienen der Massage und Bewegung und können organische und psychologische Vorteile bieten, die bereits in der Massotherapie anerkannt sind. Es kann gesehen werden, dass die vom Therapeuten angewendete Ballmassage in 4 grundlegende Manöver unterteilt ist, aber jedes Manöver hat seine Variationen. 


\section{REFERENZEN}

BERTOJA, Vanessa Gomes Bertoja; TOKARS, Eunice. Os benefícios da massagem relaxante.

em:

https://www.librarybus.com/view?t=0s+benef\%C3\%ADcios+da+massagem+relaxant e+-+TCC+On-line+-

+Tuiuti\&u=http\%3A\%2F\%2Ftcconline.utp.br\%2Fmedia\%2Ftcc\%2F2017\%2F05\%2F OS-BENEFICIOS-DA-MASSAGEM-RELAXANTE.pdf. Acesso em: 10 jan.2018.

BRAUN, Mary Beth; SIMONSON, Stephanie. Massoterapia. São Paulo: Manole, 2007.

CANNECCHIA, Marcela Cleto. Benefícios da massagem relaxante para o homem da atualidade. Pesquisa e Ação. Mogi das Cruzes, SP. v.5, n.1, p. 46-49. Jun. 2019. Disponível em: https://revistas.brazcubas.br/index.php/pesquisa/article/view/574/703. Acesso em: 05 ago. 2019.

CARVALHO, Rosemeire de Jesus; ALMEIDA, Maria Antonieta Pereira Tigre. Efeitos da Massoterapia sobre o Sistema Imunológico. Id on Line Rev. Mult. Psic. v.12, n. 40. p.353-366, 2018

CASSAR, Mario-Paul. Manual de Massagem Terapêutica: um guia completo de massoterapia para o estudante e para o terapeuta. São Paulo: Manole, 2001.

CLAY, James. H.; POUNDS, David. M. Massoterapia Clínica: integrando anatomia e tratamento. São Paulo: Manole, 2008.

ELLSWORTH, Abigail. Massagem: anatomia ilustrada, guia completo de técnicas básicas de massagem. Barueri, São Paulo: Manole, 2012.

Florentino, Danielle de M. A fisioterapia no alívio da dor: uma visão reabilitadora em cuidados paliativos. Brazilian Journal of Health and Biomedical Sciences. Rio de Janeiro; Revista do Hospital Universitário Pedro Ernesto. v.11, n.2, abr/jun, 2012. 
FRITZ, Sandy. Fundamentos da Massagem Terapêutica. 2.ed. São Paulo: Manole, 2002.

GOVERNO DO ESTADO DO CEARÁ. Técnico em Massoterapia: técnicas clássicas e modernas de massoterapia. Escola Estadual de Educação Profissional - Ensino Médio Integrado à Educação Profissional - Curso Técnico em Massoterapia. Fortaleza Ceará, 2010.

PEREIRA, Maria de Fátima Lima. Recursos Técnicos em Estética. Vol. I. Série Curso de Estética. São Paulo. Difusão Editora. 2013.

RAMOS, Andréa Rosa. Técnicas de massagem corporal. Londrina: Editora e Distribuidora Educacional S.A, 2017

SILVA, Carina Geremias dos Santos. Massoterapia Mãos de Anjo. Plano de Negócios. PG, Gravataí, v. 2, n. 2, 2016. Disponível em: http://revista.faqi.edu.br/index.php/PN/article/view/259/277. Acesso em: 02 ago 2019.

STRARKEY, Chad. Recursos Terapêuticos em Fisioterapia. 4 ed. São Paulo: Manole, 2017.

VERSAGI, Charlotte Michael. Protocolos Terapêuticos de Massoterapia: técnicas passo a passo para diversas condições clínicas. Barueri, São Paulo: Manole, 2012.

WOOD, E. C; BECKER, P. D. Técnicas de Massagem de Beard. 5. ed. Rio de Janeiro: Elsevier, 2008.

Eingereicht: August 2019.

Genehmigt: Oktober 2019. 